\title{
Individual determinants of research utilization by nurses: a systematic review update
}

Janet E Squires ${ }^{1 *}$, Carole A Estabrooks², Petter Gustavsson ${ }^{3}$, Lars Wallin ${ }^{4}$

\begin{abstract}
Background: Interventions that have a better than random chance of increasing nurses' use of research are important to the delivery of quality patient care. However, few reports exist of successful research utilization in nursing interventions. Systematic identification and evaluation of individual characteristics associated with and predicting research utilization may inform the development of research utilization interventions.

Objective: To update the evidence published in a previous systematic review on individual characteristics influencing research utilization by nurses.

Methods: As part of a larger systematic review on research utilization instruments, 12 online bibliographic databases were searched. Hand searching of specialized journals and an ancestry search was also conducted. Randomized controlled trials, clinical trials, and observational study designs examining the association between individual characteristics and nurses' use of research were eligible for inclusion. Studies were limited to those published in the English, Danish, Swedish, and Norwegian languages. A vote counting approach to data synthesis was taken.

Results: A total of 42,770 titles were identified, of which 501 were retrieved. Of these 501 articles, 45 satisfied our inclusion criteria. Articles assessed research utilization in general $(n=39)$ or kinds of research utilization $(n=6)$ using self-report survey measures. Individual nurse characteristics were classified according to six categories: beliefs and attitudes, involvement in research activities, information seeking, education, professional characteristics, and socio-demographic/socio-economic characteristics. A seventh category, critical thinking, emerged in studies examining kinds of research utilization. Positive relationships, at statistically significant levels, for general research utilization were found in four categories: beliefs and attitudes, information seeking, education, and professional characteristics. The only characteristic assessed in a sufficient number of studies and with consistent findings for the kinds of research utilization was attitude towards research; this characteristic had a positive association with instrumental and overall research utilization.

Conclusions: This review reinforced conclusions in the previous review with respect to positive relationships between general research utilization and: beliefs and attitudes, and current role. Furthermore, attending conferences/in-services, having a graduate degree in nursing, working in a specialty area, and job satisfaction were also identified as individual characteristics important to research utilization. While these findings hold promise as potential targets of future research utilization interventions, there were methodological problems inherent in many of the studies that necessitate their findings be replicated in further research using more robust study designs and multivariate assessment methods.
\end{abstract}

\footnotetext{
* Correspondence: jasquires@ohri.ca

${ }^{1}$ Clinical Epidemiology Program, Ottawa Hospital Research Institute, Ottawa,

Ontario, Canada

Full list of author information is available at the end of the article
}

\section{Biomed Central}

(C) 2011 Squires et al; licensee BioMed Central Ltd. This is an Open Access article distributed under the terms of the Creative Commons Attribution License (http://creativecommons.org/licenses/by/2.0), which permits unrestricted use, distribution, and reproduction in any medium, provided the original work is properly cited. 


\section{Background}

In this paper, we update the evidence published in a previous systematic review on individual characteristics that influence nurses' use of research evidence in clinical practice. Research utilization refers to 'that process by which specific research-based knowledge (science) is implemented in practice' [1]. In recent years, research utilization by nurses has received increased attention in the literature and has been conceptualized and measured in terms of four kinds or types of research use: instrumental, conceptual, persuasive (or symbolic), and overall [1-3]. Instrumental research utilization refers to the concrete application of research findings in clinical practice. Conceptual research utilization refers to the cognitive use of research where the research may be used to change one's thinking about a specific practice, but may or may not result in a change in action. Persuasive or symbolic research utilization is the use of research as a persuasive or political tool to legitimate a position or influence the practice of others. Overall research utilization is an omnibus construct and refers to the use of any kind of research in any way $[1,4]$.

Research utilization scholars continuously express concern about whether nurses use the best available scientific (i.e., research) evidence to guide their clinical practice [4-7]. This disparity between the availability of research evidence and its use in practice is often referred to as the 'research-practice gap.' The nature of this gap has been the subject of debate in the nursing literature. Larsen et al. [8], for example, have argued that there is no theory-practice gap; that the knowledge forms at issue in theory-practice gap discourse are radically different in kind. This stands in contrast to the views of other well-respected theorists (e.g., Allmark [9] and Fealy [10]) who articulate the nature of the gap, its origins, and in some cases, solutions to it. While, several examples of the research-practice gap have been highlighted in the nursing literature, most of the evidence is anecdotal due to difficulties surrounding attempts to measure whether or not nursing practice is researchbased [11]. It remains generally accepted however that a research-practice gap exists.

Despite increased knowledge of the benefits of adopting a research-based approach to providing nursing care and of increased availability of research findings for nurses, the use of research findings in nursing practice remains, at best, slow and haphazard [12-14]. As a result, patients frequently do not receive best (or even optimal) nursing care. In response, there is an accelerated research agenda calling for the implementation of interventions to increase research use by nurses. However, relatively few reports of research utilization interventions in nursing exist and more importantly, where they do exist, positive findings are generally not reported [15]. One review examining interventions to increase research utilization by nurses has been published. Thompson et al. [16] concluded findings on the effectiveness of interventions to increase research use in nursing are equivocal and at best, a combination of educational interventions and local opinion leaders or multidisciplinary teamwork may be effective. One reason for this relative lack of knowledge on successful research utilization interventions in nursing, we argue, is the lack of systematic identification and evaluation of factors (individual, contextual, and organizational) associated with research utilization.

In a previous systematic review of individual characteristics related to research utilization by nurses, Estabrooks et al. [17] identified 95 characteristics that they grouped into six core categories: beliefs and attitudes, involvement in research activities, information seeking, education, professional characteristics, and other socio-economic factors. The six categories were not predetermined but emerged from the data extraction. By using a vote-counting approach to synthesis, Estabrooks et al. [17] concluded the most frequently studied individual characteristic and the only one with a consistently positive effect was 'attitude towards research', which is part of the larger category 'beliefs and attitudes.' Findings for other individual characteristics were highly equivocal and were characterized by serious study design and methodological flaws. In this paper, we update the evidence on individual characteristics of research utilization by searching additional electronic databases and by adding the results of studies published between 2001 and 2008 to the evidence reported in the previous review. We also expand on the previous review by reporting on the magnitude of effect between individual nurse characteristics and research utilization and by searching for and examining literature on kinds of research utilization (i.e., instrumental, conceptual, persuasive, overall) with respect to individual characteristics important to research utilization in nursing.

\section{Methods}

\section{Selection criteria for studies Types of study}

Randomized controlled trials, clinical trials, and observational (i.e., quasi-experimental, cohort, case-control, cross-sectional) designs that examined the association between individual characteristics and nurses' use of research in practice were eligible for inclusion. Case reports and editorials were excluded. Studies were further limited to those published in the English, Danish, Swedish, and Norwegian languages. There were no restrictions on the basis of country of origin, when the study was undertaken, or publication status. 


\section{Type of participant, characteristic, and outcome}

We considered studies that examined relationships between individual characteristics and nurses' use of research. A nurse was defined as a professional who provides care in a clinical setting; this definition includes registered nurses, licensed practical nurses, nurse leaders, and clinical nurse educators. All individual characteristics, modifiable and non-modifiable, were eligible for inclusion. The outcome of interest was research utilization. We defined research utilization as the use of research-based information - that is, information that is empirically derived. This information could be reported in a primary research article, review/ synthesis report, or protocol. If the study involved the use of a protocol, the authors were required to make the research-basis for the protocol apparent in the report. We excluded articles that reported on: the adherence to clinical practice guidelines, rationale being that clinical practice guidelines can be based on nonresearch evidence (e.g., expert opinion), and the use of one specific-research-based practice if the purpose was not to examine nurses' use of research in practice generally. We did include nurses' use of protocols where the research-base of the protocol was made explicit in the research report. We also required that the relationship between the individual characteristic(s) and research utilization be expressed quantitatively (and tested statistically).

\section{Search strategy for identification of studies}

This review was conducted as part of a larger review on research utilization instruments [18]. The objectives of the larger review are: to identify instruments used to measure research utilization by healthcare providers, healthcare decision makers, and in healthcare organizations; and to assess the psychometric properties of these instruments. Research utilization instruments refer to self-report measures that assess healthcare providers' and decision makers' use of research-based knowledge in their daily practice. We searched the following 12 online bibliographic databases: Cochrane Database of Systematic Reviews (CDSR), Health and Psychosocial Instruments (HAPI), MEDLINE, CINAHL, EMBASE, Web of Science, SCOPUS, OCLC Papers First, OCLC WorldCat, Sociological Abstracts, Proquest Dissertation Abstracts, and Proquest ABI Inform. Key words and medical subject headings related to research utilization were identified prior to initiating the search. Additional File 1 displays a summary of the search strategy used in the larger review. We also hand searched the journals Implementation Science (a specialized journal in the research utilization field) and Nursing Research as well as the bibliographies of articles identified for inclusion in the review.

\section{Study identification and quality assessment}

One investigator (JES) and a research assistant screened the titles and abstracts of the articles identified by the search strategy. Articles that potentially met our inclusion criteria, or where there was insufficient information to make a decision regarding inclusion, were retrieved and assessed for relevance by one investigator (JES) and a research assistant. Disagreements throughout the selection process were resolved by consensus. To assess methodological quality of the final set of articles, we adapted two previously used tools: Estabrooks' Quality Assessment and Validity Tool for Cross-Sectional Studies, and the Quality Assessment Tool for Quantitative Studies. Each article had a quality appraisal performed by two reviewers. Articles were classified as weak, moderate-weak, moderate-strong, or strong using a system developed based on work by De Vet et al. [19] that has been used in other published systematic reviews $[17,20,21]$. All discrepancies in quality assessment were resolved through consensus.

Estabrooks' Quality Assessment and Validity Tool was developed based on the Cochrane Collaboration guidelines (in existence in 2001) and medical literature $[22,23]$. The tool contains a maximum of 16 total points covering three core domains: sample, measurement, and statistical analysis (Additional File 2). In order to derive a final score for each of the included articles (cross-sectional design), the total number of points obtained was divided by the total number of possible points, allowing for a score between 0 and 1 for each article. The articles were then classified as weak $(<0.50)$, moderate-weak ( 0.51 to 0.65$)$, moderate-strong ( 0.66 to 0.79 ), or strong (0.80 to 1.00$)$.

The Quality Assessment Tool for Quantitative Studies Tool, developed for the Canadian Effective Public Health Practice Project, has been judged suitable to be used in systematic reviews of interventions $[24,25]$. The tool contains a maximum of 18 total points covering six content areas: selection bias (is the study sample representative of the target population), allocation bias (extent that assessments of exposure and outcome are likely to be independent), confounders (were important confounders reported and appropriately managed), blinding (were the outcome assessor(s) blinded to the intervention or exposure status of participants), data collection methods (reliability and validity of data collection methods and instruments), and withdrawals and dropouts (percentage of participants completing the study) (Additional File 3). Each article is scored as weak, moderate, strong, or not applicable in each of these six areas according to preset criteria that accompany the tool. The tool developers do not provide a means for calculating an overall quality score. However, in order to compare the quality scores for each included article 
that used an intervention design (assessed with this tool) to the included articles that used cross-sectional designs (assessed with Estabrooks' Quality Assessment and Validity Tool), we derived an overall quality score for each article. To derive this score, we assigned values of 1,2 , and 3 to the categorizations of weak, moderate, and strong in each content area respectively. A final quality score for each article was then obtained by dividing the summative score obtained by the number of applicable content areas (i.e., by 6 - the number of points not applicable for the article). The articles were then classified as weak (1.0 to 2.0), moderate-weak (2.1 to 2.34$)$, moderate-strong (2.35 to 2.66$)$, or strong (2.67 to 3.0$)$.

\section{Data extraction and analysis}

One reviewer (JES) extracted data from all included articles. Extracted data was double checked by a research assistant for accuracy. Data were extracted on study design, objectives, sample and subject characteristics, theoretical framework, instruments used, reliability, validity, and key findings with respect to relationships between individual characteristics and nurses' research utilization (Tables 1 and 2 and Additional File 4). All discrepancies in data extraction were resolved through consensus.

We present the findings from this review update descriptively according to: the individual characteristics assessed, and whether research utilization was assessed as a general phenomenon or as specific kinds. We used the same six categories of individual nurse characteristics suggested in the earlier review by Estabrooks et al. (2003) for comparability: beliefs and attitudes, involvement in research activities, information seeking, education, professional characteristics, and other socioeconomic factors. A seventh category, critical thinking, emerged and is reported on in this review with respect to kinds of research utilization. Examples of the characteristics that fall within each of these categories can be seen in Tables 1 and 2 .

We used a vote-counting approach to data synthesis. That is, the overall assessment of evidence for the association between an individual characteristic and research utilization was based on the relative number of studies demonstrating, and failing to demonstrate, statistically significant associations. As recommended by Grimshaw et al. [26], we supplemented this approach by also extracting all associations showing a positive direction of effect and the magnitude of effect for statistically significant effects (regardless of direction) when it was provided in the articles. These details are presented in Tables 1 and 2. However, because of large inconsistencies in how the associations were evaluated between studies, limited conclusions on the magnitude of the associations between research utilization and specific individual characteristics could be drawn.

We developed the following set of a priori rules to guide our synthesis:

1. In order to reach a conclusion as to whether or not an individual characteristic was associated with research utilization by nurses, it had to be assessed in a minimum of four articles. Characteristics assessed in less than four articles were coded as inconsistent (i.e., insufficient evidence to reach a conclusion). There is no agreed benchmark with respect to the number of studies required to reach a conclusion concerning the relationship between two or more variables when conducting a systematic review. Within the Cochrane Collaboration, where higher levels of evidence (e.g., randomized controlled trials, pseudo-randomized controlled trials) are routinely utilized, at least one high quality study is recommended; with more studies desired. When only lower levels of evidence (e.g., non-randomised studies, observational studies) are available, no direction with respect to the number of studies required is offered [27]. A recent review [28] (utilizing observational studies) that examined the extent to which social cognitive theories (that are comprised of individual characteristics) explain healthcare professionals' intention to adopt clinical behavior used a cut-off of three studies. In this review, we set our cut-off slightly higher, at four studies, to ensure we did not draw conclusions based on occasional/random findings.

2. Characteristics that were assessed in four or more articles were coded as significant, not significant, or equivocal, depending on which of these three categories $60 \%$ or more of the articles fell within. For example, if four articles existed and two of these articles found the characteristic to be significant and two articles not significant, the characteristic was coded as equivocal.

3. Where bivariate and multivariate statistics were both offered in an article as evidence, we used the more robust multivariate findings in our synthesis to reach a conclusion as to whether or not a relationship existed between the individual characteristic(s) and research utilization.

\section{Results}

\section{Description of studies}

Figure 1 summarizes article selection for this review. The database and hand searches yielded 42,770 titles and abstracts. Of these 42,770 articles, 501 were identified as being potentially relevant after a title and 
Table 1 Summary of findings for studies reporting research utilization in general ( $\mathbf{n}=\mathbf{3 9}$ articles)

\begin{tabular}{|c|c|c|c|c|}
\hline Individual Determinant & First Author & Significance* & Direction and Magnitude & Comment \\
\hline \multicolumn{5}{|l|}{ 1. BELIEFS AND ATTITUDES } \\
\hline Perceived support for research & Butler [71] & NS & & \\
\hline \multirow[t]{8}{*}{ Attitude toward research } & Champion [50] & S & $+(r=.55)$ & \\
\hline & Estabrooks [31] & S & + LISREL & $\begin{array}{l}\text { Chi square }=55.91 p=.263 \text { for model with } \\
\text { attitude, belief suspension and in-services }\end{array}$ \\
\hline & Hatcher [52] & S & $+(r=.65-.82)$ & \\
\hline & Lacey [54] & S & $+(r=.674)$ & \\
\hline & Prin [56] & S & $+(r=.58)$ & \\
\hline & Tranmer [57] & S & $+(\beta=.64)$ & \\
\hline & Varcoe [61] & S & $+(r=.41)$ & $\begin{array}{l}\text { S for general research use (RUQ); NS for } \\
\text { specific practices }\end{array}$ \\
\hline & Wells [72] & S & $+(\beta=1.62)$ & \\
\hline $\begin{array}{l}\text { Expectation of self to use } \\
\text { research }\end{array}$ & Varcoe [61] & S & $+(r=.51)$ & $\begin{array}{l}\text { With general use of research (not specific } \\
\text { findings) }\end{array}$ \\
\hline Expressed interest in research & Varcoe [61] & S & $+(r=.50)$ & $\begin{array}{l}\text { With general use of research (not specific } \\
\text { findings) }\end{array}$ \\
\hline Problem solving ability & Estabrooks [31] & NS & & \\
\hline \multirow[t]{2}{*}{ Cosmopoliteness } & Estabrooks [31] & NS & & \\
\hline & Estabrooks [31] & NS & & \\
\hline \multirow[t]{2}{*}{ Autonomy } & Forbes [62] & S & $+(r=0.08)$ & \\
\hline & McCloskey [33] & S & $+(\beta=0.135)$ & \\
\hline Dogmatism & Estabrooks [31] & NS & & \\
\hline Activism & Estabrooks [31] & NS & & \\
\hline Belief suspension & Estabrooks [31] & S & + (LISREL) & $\begin{array}{l}\text { Chi square }=55.91 p=.263 \text { for model with } \\
\text { attitude, in-services, belief suspension }\end{array}$ \\
\hline Theoretical orientation & Estabrooks [31] & NS & & \\
\hline Trust & Estabrooks [31] & NS & & \\
\hline Confidence & Wells [72] & NS & & $\begin{array}{l}\text { Confidence in research related activities (e.g., } \\
\text { reading research, discussing research) }\end{array}$ \\
\hline Career commitment & Stiefel [60] & S & $+\left(R^{2}=0.13\right)$ & MANOVA \\
\hline $\begin{array}{l}\text { Perception of nurse as a RU } \\
\text { barrier }\end{array}$ & Bostrom [51] & S & $+(t=2.512)$ & Research user reports less individual barriers \\
\hline Awareness (overall) of practice & Squires [14] & S & $+(\beta=2.52)$ & For 'user of research' \\
\hline $\begin{array}{l}\text { Awareness of practice by } \\
\text { regular use }\end{array}$ & Squires [14] & S & $+(\beta=3.49)$ & For 'user of research' \\
\hline Research awareness & Wells [72] & NS & & \\
\hline $\begin{array}{l}\text { Persuaded (believe in) of the } \\
\text { practice }\end{array}$ & Squires [14] & S & $+(\beta=2.11)$ & For 'user of research' \\
\hline
\end{tabular}

\section{INVOLVEMENT IN RESEARCH ACTIVITIES}

\begin{tabular}{|c|c|c|c|c|}
\hline $\begin{array}{l}\text { Current data collection for } \\
\text { others }\end{array}$ & Butler [71] & S & $+(\mathrm{OR}=4.04)$ & \\
\hline \multirow{2}{*}{$\begin{array}{l}\text { Participation in research-related } \\
\text { activities }\end{array}$} & Berggren [46] & NS & & \\
\hline & McCleary [29] & S & + & Test statistic not given \\
\hline $\begin{array}{l}\text { Participation in research as } \\
\text { subject }\end{array}$ & Hatcher [52] & NS & & \\
\hline Past use of research & Butler [71] & $S$ & $+O R=20.0$ & \\
\hline Job related research activities & Rutledge [49] & $S$ & $+(r=.0673$ to .1272$)$ & 5 for 3 of 8 practices \\
\hline \multirow[t]{2}{*}{ Participation in research study } & Brett [44] & NS & & \\
\hline & Nash [55] & NS & & \\
\hline $\begin{array}{l}\text { Education for research } \\
\text { participation }\end{array}$ & Logsdon [77] & $S$ & $+(r=.32)$ & \\
\hline
\end{tabular}


Table 1 Summary of findings for studies reporting research utilization in general ( $\mathbf{n}=\mathbf{3 9}$ articles) (Continued)

\begin{tabular}{|c|c|c|c|c|}
\hline Research participation & Tsai [74] & S & $+(r=.3268)$ & \\
\hline $\begin{array}{l}\text { Involvement in research } \\
\text { projects }\end{array}$ & Tranmer [57] & NS & & \\
\hline Research experience & Varcoe [61] & $\mathrm{S}$ & $+(r=.37)$ & $\begin{array}{l}\text { With general use of research (not specific } \\
\text { findings) }\end{array}$ \\
\hline $\begin{array}{l}\text { Participation in quality } \\
\text { management }\end{array}$ & McCleary [29] & S & + & Test statistic not given \\
\hline $\begin{array}{l}\text { Participation in quality } \\
\text { improvement }\end{array}$ & Wallin [58] & S & $+\left(X^{2}=11.1\right)$ & \\
\hline $\begin{array}{l}\text { Completion of the research } \\
\text { study }\end{array}$ & Tsai [75] & NS & & \\
\hline \multicolumn{5}{|l|}{ 3. INFORMATION SEEKING } \\
\hline Nursing texts as information & Barta [45] & NS & & \\
\hline $\begin{array}{l}\text { Nursing journals as information } \\
\backslash\end{array}$ & Barta [45] & $S$ & $+(\mathrm{t}=-2.36)$ & \\
\hline Education by specialty groups & Barta [45] & NS & & \\
\hline $\begin{array}{l}\text { Personal experience as } \\
\text { information }\end{array}$ & Squires [14] & S & $+(\beta=0.55)$ & For 'consistent research user' \\
\hline P\&P manual as information & Squires [14] & NS & & \\
\hline $\begin{array}{l}\text { In-services as a source of } \\
\text { knowledge }\end{array}$ & Squires [14] & NS & & \\
\hline Attended education program & Berggren [46] & NS & & \\
\hline Critical reading skills & Tranmer [57] & S & $+(\beta=0.19)$ & Pre-test \& Post-test respondents combined \\
\hline Use computer & Wallin [69] & S & $+(\beta=0.142)$ & \\
\hline Time per week on the internet & Wallin [69] & NS & & \\
\hline Internet use & Cummings [68] & NS & & \\
\hline Have a personal computer & Wallin [69] & NS & & \\
\hline \multicolumn{5}{|l|}{ Reading activities Read journals } \\
\hline & Berggren [46] & NS & & \\
\hline \multirow[t]{3}{*}{ Hours reading journals } & Brett [44] & $S$ & $+(r=.163)$ & \\
\hline & Coyle [47] & NS & & \\
\hline & Michel [48] & NS & & \\
\hline \multirow[t]{3}{*}{ Number of journals read } & Rodgers [12] & S & $+(Z=2.98)$ & \\
\hline & Rutledge [49] & S & $+(r=.0901)$ & 1 of 8 practices \\
\hline & Wells [72] & NS & & \\
\hline \multirow[t]{2}{*}{ Reads Heart \& Lung } & Coyle [47] & S & $+\left(X^{2}=3.795\right)$ & \\
\hline & Michel [48] & S & + Mann Whitney $U=1422.0$ & \\
\hline \multirow[t]{2}{*}{ Reads Nursing Research } & Brett [44] & $S$ & $+\left(X^{2}=12.422\right)$ & \\
\hline & Michel [48] & NS & & \\
\hline Reads $R N$ & Brett [44] & S & $+\left(X^{2}=8.925\right)$ & \\
\hline \multirow{5}{*}{$\begin{array}{l}\text { Attendance at conferences/in- } \\
\text { services }\end{array}$} & Butler [71] & NS & & \\
\hline & Coyle [47] & S & $+\left(X^{2}=5.179\right)$ & To total TIAB score \\
\hline & Estabrooks [31] & S & $+($ LISREL $)$ & $\begin{array}{l}\text { Chi square }=55.91 p=.263 \text { for model with } \\
\text { attitude, belief suspension and in-services }\end{array}$ \\
\hline & Michel [48] & S & + Mann Whitney $U=1291.5$ & \\
\hline & Rutledge [49] & S & $+(r=.1168)$ & All 8 practices combined \\
\hline \multirow[t]{2}{*}{ Hours of continuing education } & Brett [44] & NS & & \\
\hline & Coyle [47] & NS & & \\
\hline $\begin{array}{l}\text { Number of study days } \\
\text { attended }\end{array}$ & Rodgers [12] & S & $+(r=.095)$ & \\
\hline Time spent studying (on duty) & Rodgers [12] & NS & & \\
\hline
\end{tabular}


Table 1 Summary of findings for studies reporting research utilization in general ( $\mathbf{n}=\mathbf{3 9}$ articles) (Continued)

\begin{tabular}{|c|c|c|c|c|}
\hline Time spent studying (off duty) & Rodgers [12] & $S$ & $+(r=.1)$ & \\
\hline MEDLINE usage & Prin [56] & S & $+(r=.2526)$ & \\
\hline \multicolumn{5}{|l|}{ 4. EDUCATION } \\
\hline $\begin{array}{l}\text { Increasing levels (multiple } \\
\text { levels: diploma, bachelors, } \\
\text { masters, PhD; post-hoc analysis } \\
\text { not provided) }\end{array}$ & Brett [44] & NS & & Diploma, Bachelors, Masters \\
\hline
\end{tabular}
not provided)

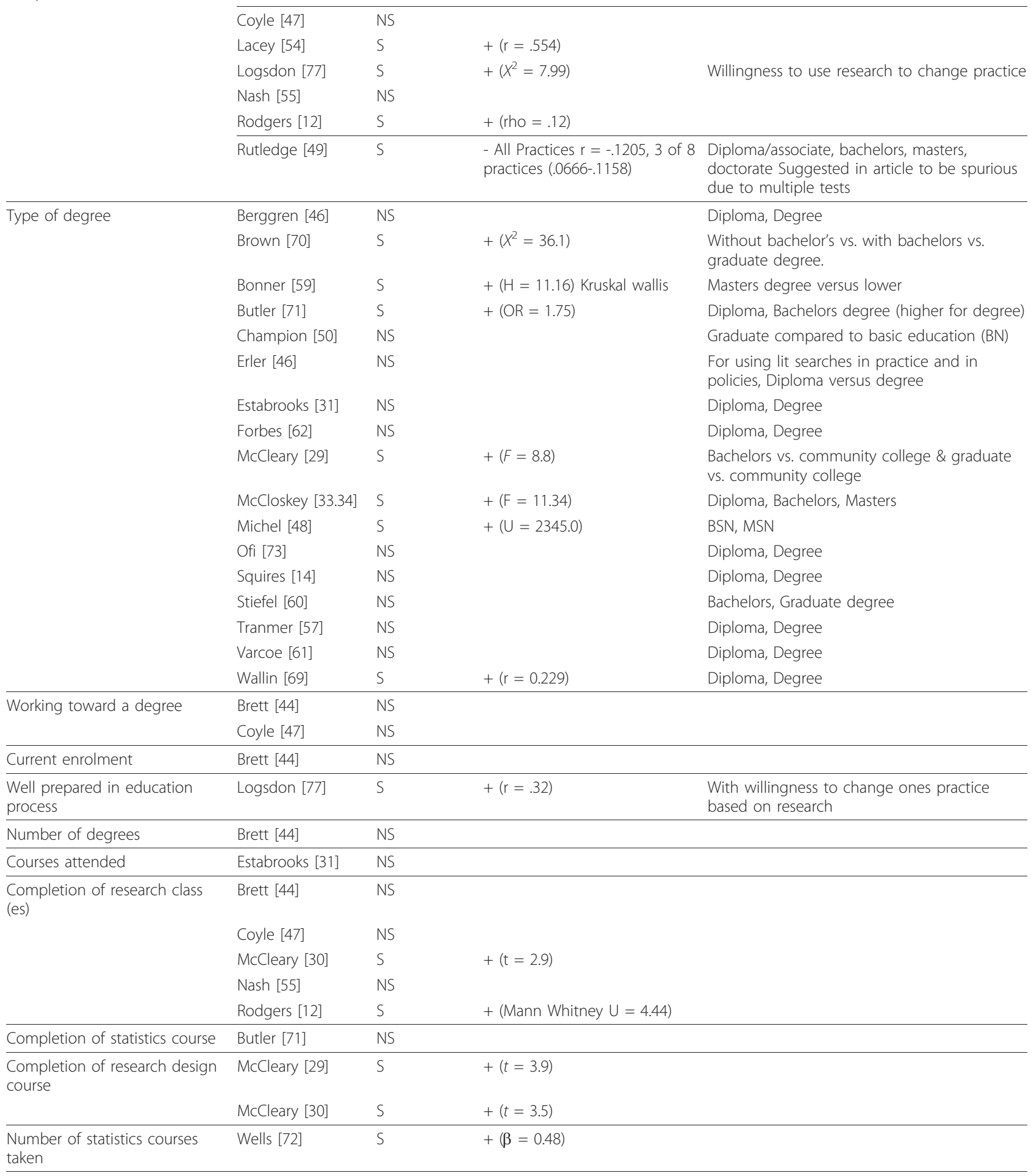


Table 1 Summary of findings for studies reporting research utilization in general $(\mathbf{n}=\mathbf{3 9}$ articles) (Continued)

\begin{tabular}{llll}
\hline Years since basic education & Brett [44] & NS & \\
\hline Years since last degree & Estabrooks [31] & NS & \\
\hline $\begin{array}{l}\text { Taught a topic based on } \\
\text { research }\end{array}$ & Rodgers [12] & S & + (Mann Whitney U = 4.93) \\
\hline Having project 2000 training & Parahoo [35] & NS & \\
\hline
\end{tabular}

\section{PROFESSIONAL CHARACTERISTICS}

\begin{tabular}{|c|c|c|c|c|}
\hline \multirow[t]{2}{*}{ Full or part-time status } & \multirow{2}{*}{$\begin{array}{l}\text { Butler [71] } \\
\text { Wallin [69] }\end{array}$} & \multicolumn{3}{|l|}{ NS } \\
\hline & & $S$ & $+(\beta=0.228)$ & For work full time \\
\hline \multirow[t]{12}{*}{ Years employed as an RN } & Butler [71] & NS & & \\
\hline & Champion [50] & NS & & \\
\hline & Coyle [47] & NS & & \\
\hline & Estabrooks [31] & NS & & \\
\hline & McCleary [29] & NS & & \\
\hline & McCloskey [34] & NS & & \\
\hline & Michel [48] & NS & & \\
\hline & Rodgers [12] & NS & & \\
\hline & Squires [14] & S & $+(\beta=0.07)$ & For 'consistent research user' \\
\hline & Stiefel $[60]$ & $S$ & $+(r=.22)$ & \\
\hline & Tranmer [57] & NS & & \\
\hline & Wallin [69] & NS & & \\
\hline Years in post (hospital) & Tranmer [57] & NS & & \\
\hline \multirow[t]{9}{*}{ Current role } & Berggren [46] & NS & & Staff midwife or midwifery sister \\
\hline & Bonner [59] & $S$ & + Kruskal Wallis $(H=12.67)$ & $\begin{array}{l}\text { Nurse unit managers and consultant report } \\
\text { more use than staff nurses }\end{array}$ \\
\hline & Butler [71] & S & $+(\mathrm{OR}=5.01)$ & $\begin{array}{l}\text { Those in leadership or advanced roles report } \\
\text { more use than staff nurses }\end{array}$ \\
\hline & Connor [66] & NS & & \\
\hline & Hatcher [52] & $S$ & $+(t=5.57)$ & $\begin{array}{l}\text { Those in leadership of advanced roles report } \\
\text { more use as compared to staff nurses }\end{array}$ \\
\hline & McCloskey $[33,34]$ & S & $+(F=7.901)$ & $\begin{array}{l}\text { Management position or advanced practice } \\
\text { nurses vs. staff nurses }\end{array}$ \\
\hline & Rodgers [12] & NS & & Charge nurse vs. staff nurse \\
\hline & Wallin [69] & S & $-(\beta=-0.395)$ & $\begin{array}{l}\text { Staff nurse versus other (staff nurses use less } \\
\text { research) }\end{array}$ \\
\hline & Wells [72] & NS & & Staff nurse, nurse manager \\
\hline \multirow[t]{9}{*}{ Clinical specialty } & Estabrooks [31] & NS & & \\
\hline & Michel $[48]$ & NS & & \\
\hline & Forbes [62] & S & + ANOVA $(F=5.370$ & $\begin{array}{l}\text { Higher RU for critical care nurses as compared } \\
\text { to medical/surgical or obstetrical/ } \\
\text { gynecological }\end{array}$ \\
\hline & Humphris [53] & S & $+X^{2}$ (test value not reported) & $\begin{array}{l}\text { Greater number of diabetic nurse specialists } \\
\text { implement specific findings into practice as } \\
\text { compared to the non-nurse specialist group }\end{array}$ \\
\hline & Nash [55] & $S$ & $+\operatorname{ANOVA}(F=2.35)$ & $\begin{array}{l}\text { Area worked (highest RU mean to lowest): } \\
\text { Education, other, hospital inpatient, outpatient } \\
\text { clinic, office }\end{array}$ \\
\hline & Parahoo [36] & S & $+\left(X^{2}=3.79\right)$ & Medical vs. surgical nurses \\
\hline & Squires [14] & S & $-(\beta=-0.42)$ & $\begin{array}{l}\text { Med-surg compared to critical care unit (med- } \\
\text { surg use less than CC) }\end{array}$ \\
\hline & Stiefel $[60]$ & S & $\begin{array}{l}+(\text { Wilk's lambda }=0.76, F= \\
2.23)\end{array}$ & $\begin{array}{l}\text { Critical care higher RU than medicine, surgery, } \\
\text { oncology }\end{array}$ \\
\hline & Wright [78] & NS & & $\begin{array}{l}\text { Analyzed groups by practice area (general } \\
\text { hospital, psychiatric hospital, or community } \\
\text { mental health) }\end{array}$ \\
\hline Number of memberships held & Coyle [47] & NS & & \\
\hline Oncology nursing society status & Rutledge [49] & S & - 2 of 8 practices $(-.068,-.080)$ & \\
\hline
\end{tabular}


Table 1 Summary of findings for studies reporting research utilization in general $(\mathbf{n}=\mathbf{3 9}$ articles) (Continued)

\begin{tabular}{|c|c|c|c|c|}
\hline Oncology certification & Rutledge [49] & NS & & \\
\hline CFRN certification & Erler [76] & $S$ & $\begin{array}{l}+\left(X^{2}=9.6-\text { use research }\right. \\
\text { literature); }\left(X^{2}=11.2-\text { translate }\right. \\
\text { findings into policies and } \\
\text { procedures })\end{array}$ & \\
\hline \multirow[t]{5}{*}{ Job satisfaction } & Coyle [47] & S & $+(r=.18)$ & \\
\hline & Estabrooks [31] & NS & & \\
\hline & Berggren [46] & NS & & \\
\hline & Forbes [62] & S & $+(r=0.13)$ & \\
\hline & Wallin [69] & S & $+(\beta=0.264)$ & \\
\hline Emotional exhaustion & Cummings [68] & S & $\begin{array}{l}\text { - (magnitude varied by } \\
\text { context) }\end{array}$ & $\begin{array}{l}\text { Coefficients significant but model not. High } \\
\text { context estimated effect }=-.109 ; \text { partially high } \\
\text { context estimated effect }=-.191 \text {; partially low } \\
\text { context estimated effect }=-.334 \text {; low context } \\
\text { estimated effect }=-.251\end{array}$ \\
\hline \multirow[t]{2}{*}{ Stress } & Forbes [62] & S & $-(r=-0.13)$ & $\begin{array}{l}\text { Personal job stress: Juggling expectations of } \\
\text { other professionals and of clients }\end{array}$ \\
\hline & Forbes [62] & S & $-(r=-0.08)$ & $\begin{array}{l}\text { Situational job stress: Issues such as } \\
\text { equipment, time, and staffing }\end{array}$ \\
\hline Affiliation & Estabrooks [31] & NS & & \\
\hline Dependant care hours & Estabrooks [31] & NS & & \\
\hline \multirow[t]{2}{*}{ Hours/week worked } & Estabrooks [31] & NS & & \\
\hline & Wallin [69] & NS & & \\
\hline Shift usually worked & Estabrooks [31] & NS & & \\
\hline Shift satisfaction & Estabrooks [31] & NS & & \\
\hline National certification & Stiefel [60] & NS & & \\
\hline
\end{tabular}

\section{SOCIO-DEMOGRAPHIC AND SOCIO-ECONOMIC FACTORS}

\begin{tabular}{lll}
\hline Age & Berggren [46] & NS \\
& Butler [71] & NS \\
& Champion [50] & NS \\
& Cummings [68] & NS \\
& Estabrooks [31] & NS \\
& Lacey [54] & NS \\
& McCleary [29] & NS \\
& Rodgers [12] & NS \\
& Wallin [69] & NS \\
\hline Married or partnered/Marital & Estabrooks [31] & NS \\
status & Estabrooks [31] & NS \\
\hline Family income & Estabrooks [31] & NS \\
\hline Health/lifestyle activity & Estabrooks [31] & NS \\
\hline Gender & Stiefel [60] & NS \\
\hline & Wallin [69] & NS \\
\hline
\end{tabular}

*Significance: $\mathrm{NS}=$ not significant, $\mathrm{S}=$ significant at $\mathrm{p}<0.05$

abstract review. A total of 456 articles were excluded for not meeting our inclusion criteria, leaving 45 articles for inclusion in this review, and 31 (69\%) of these articles are additions to the previous review). The 45 articles represent 41 original studies; four studies have two reports each: McCleary and Brown [29,30]; Estabrooks [31,32]; McCloskey [33,34]; and Parahoo [35,36]. A list of all $(n=45)$ included articles can be found in
Additional File 4. The original review [17] included 22 articles. This review update excluded eight of these articles, leaving 14 of the original articles in the update. The eight articles were excluded for one of three reasons: they did not include a measure of research utilization as we defined it for this review update $(n=5)$ [37-41], they did not report on individual characteristics $(\mathrm{n}=2$, these two articles represented a second report of 
Table 2 Summary of findings for studies reporting kinds of research utilization ( $\mathrm{n}=\mathbf{6}$ articles)

\begin{tabular}{|c|c|c|c|c|c|}
\hline \multirow[t]{2}{*}{ Individual Determinant } & \multirow{2}{*}{$\begin{array}{l}\text { First } \\
\text { Author }\end{array}$} & \multicolumn{4}{|c|}{ Significance* (Direction and magnitude) } \\
\hline & & $\begin{array}{l}\text { Instrumental Research } \\
\text { Utilization }\end{array}$ & $\begin{array}{l}\text { Conceptual } \\
\text { Research } \\
\text { Utilization }\end{array}$ & $\begin{array}{l}\text { Persuasive } \\
\text { Research } \\
\text { Utilization }\end{array}$ & Overall Research Utilization \\
\hline \multicolumn{6}{|l|}{ 1. BELIEFS AND ATTITUDES } \\
\hline & Connor [66] & NS & NS & NS & $S+(\beta=0.234)$ \\
\hline \multirow[t]{3}{*}{ Attitude toward research } & $\begin{array}{l}\text { Estabrooks } \\
{[32]}\end{array}$ & $\begin{array}{l}\text { Canadian }-\mathrm{S}+(\mathrm{OR}= \\
\text { 1.17) US Military - NS }\end{array}$ & Not assessed & Not assessed & $\begin{array}{l}\text { Canadian }-\mathrm{S}+(\mathrm{OR}=1.21) \mathrm{US} \\
\text { Military }-\mathrm{S}+(\mathrm{OR}=1.16)\end{array}$ \\
\hline & Kenny [63] & $S+(\beta$ not reported $)$ & NS & NS & NS \\
\hline & Milner [67] & $S+(\beta=0.120)$ & NS & $S+(\beta=0.075)$ & $S+(\beta=0.098)$ \\
\hline Importance of access to research & Kenny [63] & NS & NS & $\begin{array}{l}S+(\beta \text { not } \\
\text { reported })\end{array}$ & NS \\
\hline Cosmopoliteness & Milner [67] & NS & NS & NS & NS \\
\hline $\begin{array}{l}\text { Localite (orientation within one's } \\
\text { immediate social context) }\end{array}$ & Milner [67] & NS & $S+(\beta=0.031)$ & NS & NS \\
\hline $\begin{array}{l}\text { Interest or organizational groups } \\
\text { belonged to }\end{array}$ & Kenny [63] & NS & NS & NS & $S+(\beta$ not reported $)$ \\
\hline Adoptiveness & Milner [67] & NS & NS & NS & NS \\
\hline \multirow[t]{3}{*}{ Belief suspension } & $\begin{array}{l}\text { Estabrooks } \\
{[32]}\end{array}$ & $\begin{array}{l}\text { Canadian - NS US } \\
\text { Military - } S+(O R=1.11)\end{array}$ & Not assessed & Not assessed & $\begin{array}{l}\text { Canadian }-\mathrm{S}+(\mathrm{OR}=1.07) \mathrm{US} \\
\text { Military }-\mathrm{S}+(\mathrm{OR}=1.08)\end{array}$ \\
\hline & Kenny [63] & $S+(\beta$ not reported $)$ & NS & NS & NS \\
\hline & Connor [66] & NS & NS & NS & NS \\
\hline \multirow[t]{2}{*}{ Trust } & $\begin{array}{l}\text { Estabrooks } \\
{[32]}\end{array}$ & NS & Not assessed & Not assessed & $\begin{array}{l}\text { Canadian - NS US Military - S } \\
+(\mathrm{OR}=1.12)\end{array}$ \\
\hline & Kenny [63] & NS & NS & $\begin{array}{l}S+(\beta \text { not } \\
\text { reported })\end{array}$ & NS \\
\hline Research awareness & Milner [67] & $S+(\beta=0.037)$ & NS & $S+(\beta=0.076)$ & $S+(\beta=0.063)$ \\
\hline $\begin{array}{l}\text { Importance of various factors to } \\
\text { decision-making }\end{array}$ & Kenny [63] & NS & $\begin{array}{l}S+(\beta \text { not } \\
\text { reported })\end{array}$ & $\begin{array}{l}S+(\beta \text { not } \\
\text { reported })\end{array}$ & NS \\
\hline \multicolumn{6}{|c|}{ 2. INVOLVEMENT IN RESEARCH ACTIVITIES } \\
\hline Research involvement & Milner [67] & $S+(\beta=0.142)$ & NS & $S+(\beta=0.170)$ & $S+(\beta=0.176)$ \\
\hline \multicolumn{6}{|l|}{ 3. INFORMATION SEEKING } \\
\hline \multirow[t]{2}{*}{ Number of nursing journals read } & Connor [66] & NS & NS & NS & NS \\
\hline & Kenny [63] & NS & NS & NS & NS \\
\hline \multirow[t]{3}{*}{ Sources of knowledge } & Connor [66] & NS & NS & NS & NS \\
\hline & $\begin{array}{l}\text { Estabrooks } \\
{[32]}\end{array}$ & NS & Not assessed & Not assessed & NS \\
\hline & Kenny [63] & NS & NS & NS & NS \\
\hline Mass media & Milner [67] & NS & NS & $S+(\beta=0.194)$ & NS \\
\hline Number of journals read & Kenny [63] & $S+(\beta$ not reported $)$ & NS & NS & NS \\
\hline $\begin{array}{l}\text { Number of continuing education } \\
\text { sessions }\end{array}$ & Connor [66] & NS & $\begin{array}{l}S+(\beta \text { not } \\
\text { reported })\end{array}$ & $\begin{array}{l}S+(\beta \text { not } \\
\text { reported })\end{array}$ & $S+(\beta$ not reported $)$ \\
\hline \multirow[t]{2}{*}{ In-services attended } & Connor [66] & NS & NS & NS & NS \\
\hline & $\begin{array}{l}\text { Estabrooks } \\
{[32]}\end{array}$ & NS & Not Assessed & Not Assessed & $\begin{array}{l}\text { Canadian }-\mathrm{S}+(\mathrm{OR}=1.03) \text { US } \\
\text { Military - NS }\end{array}$ \\
\hline \multicolumn{6}{|l|}{ 4. EDUCATION } \\
\hline \multirow[t]{2}{*}{ Increasing levels } & Kenny [63] & NS & NS & NS & NS \\
\hline & Connor [66] & NS & NS & NS & NS \\
\hline Type of degree & $\begin{array}{l}\text { Estabrooks } \\
{[32]}\end{array}$ & NS & Not Assessed & Not Assessed & NS \\
\hline Possessing a degree & Milner [67] & NS & NS & NS & NS \\
\hline
\end{tabular}


Table 2 Summary of findings for studies reporting kinds of research utilization ( $\mathbf{n}=\mathbf{6}$ articles) (Continued)

\begin{tabular}{|c|c|c|c|c|c|}
\hline \multicolumn{6}{|c|}{ 5. PROFESSIONAL CHARACTERISTICS } \\
\hline \multirow[t]{2}{*}{ Years employed as an RN } & $\begin{array}{l}\text { Estabrooks } \\
{[32]}\end{array}$ & $\begin{array}{l}\text { Canadian - NS US } \\
\text { military - } S+(O R=0.97)\end{array}$ & Not Assessed & Not Assessed & NS \\
\hline & Kenny [63] & NS & NS & NS & NS \\
\hline Length of time at job title & Connor [66] & NS & NS & NS & NS \\
\hline \multirow[t]{2}{*}{ Years in post (hospital) } & Kenny [63] & NS & NS & NS & NS \\
\hline & Connor [66] & NS & NS & NS & NS \\
\hline \multirow[t]{6}{*}{ Current role } & Milner $[67]^{1}$ & NS & NS & NS & $S-(\beta=-0.265)$ \\
\hline & Milner $[67]^{2}$ & NS & $S-(\beta=-0.382)$ & $S-(\beta=-0.345)$ & NS \\
\hline & Kenny [63] & NS & NS & NS & NS \\
\hline & Connor [66] & NS & NS & NS & NS \\
\hline & Kenny [63] & NS & NS & NS & NS \\
\hline & Connor [66] & NS & NS & NS & NS \\
\hline Number of memberships held & Connor [66] & NS & NS & NS & NS \\
\hline \multicolumn{6}{|c|}{ 6. SOCIO-DEMOGRAPHIC AND SOCIO-ECONOMIC FACTORS } \\
\hline \multirow[t]{2}{*}{ Age } & Milner [67] & NS & NS & NS & $S-(\beta=-0.011)$ \\
\hline & $\begin{array}{l}\text { Profetto- } \\
\text { McGrath } \\
{[64]}\end{array}$ & NS & NS & NS & NS \\
\hline \multirow[t]{2}{*}{ Gender } & Connor [66] & NS & NS & NS & NS \\
\hline & $\begin{array}{l}\text { Estabrooks } \\
{[32]}\end{array}$ & NS & Not assessed & Not Assessed & NS \\
\hline \multicolumn{6}{|l|}{ 7. CRITICAL THINKING } \\
\hline \multirow[t]{2}{*}{$\begin{array}{l}\text { Critical thinking skills (total CCTDI } \\
\text { score) }\end{array}$} & $\begin{array}{l}\text { Profetto- } \\
\text { McGrath } \\
\text { [64] }\end{array}$ & $S+(r=.240$ & $S+(r=.27)$ & $\mathrm{S}+(r=.17)$ & $\mathrm{S}+(r=.35)$ \\
\hline & $\begin{array}{l}\text { Profetto- } \\
\text { McGrath } \\
{[65]}\end{array}$ & $\mathrm{S}+(r=.222)$ & $\mathrm{S}+(r=.205)$ & $\mathrm{S}+(r=.237)$ & $\mathrm{S}+(r=.146)$ \\
\hline
\end{tabular}

*Significance: NS = not significant, $\mathrm{S}=$ significant at $\mathrm{p}<0.05$.

1 Managers vs educators.

${ }^{2}$ RNs vs educators.

a study that did not report individual characteristics the first report of each study, which did report on individual characteristics, were included) [2,42], or did not provide a quantitative (statistical) test of the association between the individual characteristic(s) and research utilization $(n=1)$ [43].

A variety of self-report instruments, multi-item and single item, were used to measure research utilization in the 45 included articles. Multi-item instruments used included: the Nurses Practice Questionnaire $(\mathrm{n}=8)$ [12,14,44-49]; the Research Utilization Questionnaire $(\mathrm{n}=11)$ [33,34,50-58]; the Edmonton Research Orientation Survey $(\mathrm{n}=3)[29,30,59]$; and three research utilization indexes, each used in a single study [60-62]. Single-item instruments used included: Estabrooks Kinds of Research Utilization Items $(\mathrm{n}=9)$ [31,32,63-69]; Parahoo's Item $(\mathrm{n}=2)$ [35,36]; Past, Present, and Future Use Items $(n=3)$ [70-72]; and other single items, each used in a single study $(\mathrm{n}=6)$ [73-78]. The majority of articles examined research utilization by nurses in the United States $(\mathrm{n}=18,40 \%)$ followed by
Canada ( $\mathrm{n}=14,31 \%)$, Europe $(\mathrm{n}=8,18 \%)$, Australia $(\mathrm{n}=2,4.5 \%)$, China $(\mathrm{n}=2,4.5 \%)$, and Africa $(\mathrm{n}=1$, $2 \%$ ). The most commonly reported setting was hospitals ( $\mathrm{n}=28,62 \%$ ) followed by a mixture of settings, e.g., sampling from a provincial or state nursing roster ( $\mathrm{n}=$ $13,29 \%)$, nursing homes $(\mathrm{n}=2,4.5 \%)$, an educational setting $(\mathrm{n}=1,2 \%)$, and a flight team setting $(\mathrm{n}=1,2 \%)$. With respect to year of publication, the vast majority of articles were published since 1995 ( $\mathrm{n}=40,89 \%$ ). Further details on the characteristics of the included articles can be found in Additional File 4.

\section{Methodological quality of included studies}

Methodological quality of the articles included in this review is reported in Additional Files 2 and 3. All articles used an observational design: the majority $(n=43,96 \%)$ used a cross-sectional design while two articles (4\%) used a quasi-experimental design. Of the 45 included articles, one (2\%) was rated as strong, $13(29 \%)$ as moderatestrong, $18(40 \%)$ as moderate-weak, and $13(29 \%)$ as weak. Discrepancies in quality assessment related mainly 


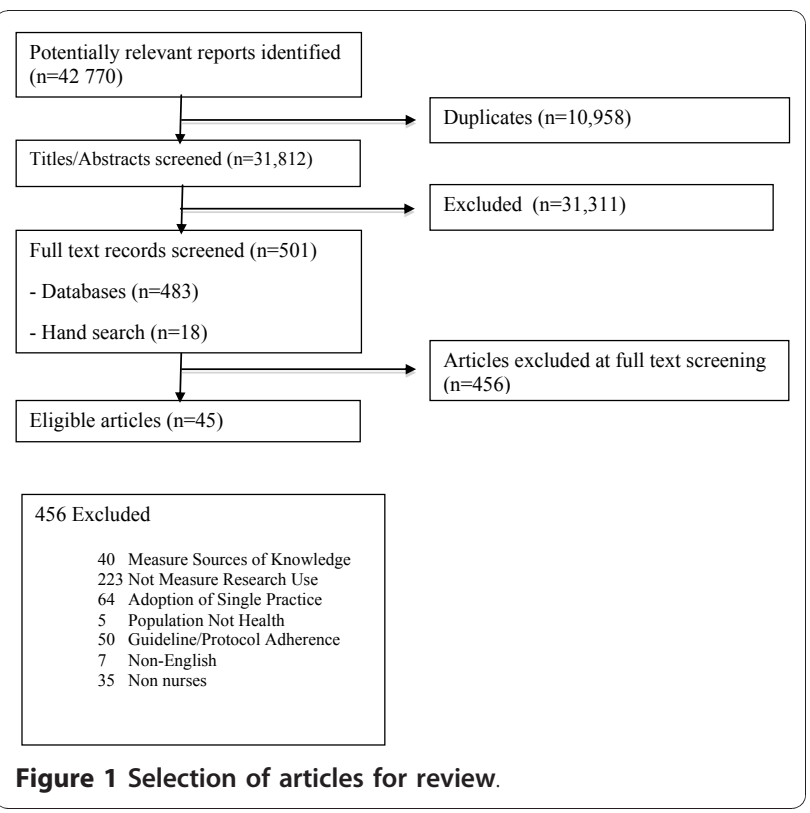

to sample representativeness, treatment of missing data, and appropriateness of the statistical test(s) used.

\section{The outcome: individual characteristics and research utilization}

Data on individual characteristics were extracted into the original six categories from the previous review [17]: beliefs and attitudes, involvement in research activities, information seeking, education, professional characteristics, socio-demographic and socio-economic factors (relabeled from other socio-economic factors), and one additional category, and critical thinking. Relationships between these characteristics and research utilization in general, and kinds of research utilization, are summarized next with additional details presented in Tables 1 and 2 respectively.

\section{Research utilization in general}

A total of 39 (87\%) articles examined relationships between individual characteristics and nurses' research utilization in general (Table 1).

\section{Beliefs and attitudes}

Fourteen articles assessed one or more individual characteristic in the beliefs and attitudes category. Of these 14 articles, six were rated as weak methodologically, five were rated as moderate-weak, and three were rated as moderate-strong (Additional Files 2 and 3). Sample sizes varied from a low of 20 participants [54] to a high of 1,117 participants [62] (Additional File 4). The most frequently assessed characteristic in this category was attitude towards research, assessed in eight articles. The majority of these eight articles were rated as weak $(\mathrm{n}=$
3) or moderate-weak ( $n=4)$ methodologically while one article received a quality rating of moderate-strong (Additional Files 2 and 3). In all eight articles, attitude towards research was measured using multi-item summated scales. A 21-item scale developed by Champion and Leach [50] with items tapping nurses' feelings about incorporating research into practice was used in four of the eight articles $[50,52,56,57]$. Similar multi-item measures, with six [31,61], 12 [54] and 15 items [72] were used in the remaining four studies. A positive association with research utilization, at statistically significant levels, was found in all eight articles. The magnitude of effect, on average, was high moderate, with correlation coefficients ranging from 0.41 to 0.82 . Other belief and attitudinal characteristics were assessed in less than four articles and therefore their results cannot be considered with any confidence.

\section{Involvement in research activities}

Thirteen articles assessed one or more individual characteristic related to nurses' involvement in research activities. Of these articles, three were rated as weak methodologically, eight were rated as moderate-weak, and two were rated as moderate-strong (Additional Files 2 and 3). Sample sizes also varied from a low of 82 participants [55] to a high of 1,100 participants [49] (Additional File 4). Examples of activities assessed that were reflective of involvement in research activities included: participation in a research study [44,55], participation in quality improvement initiatives [58], participation in quality management [29], and data collection for others conducting research [71]. Additional examples can be found in Table 1. A total of 13 individual characteristics were identified in this category overall. However, each characteristic was assessed in less than four articles, precluding us from drawing conclusions on the relationships between individual characteristics typical of involvement in research activities and nurses' use of research findings in practice.

\section{Information-seeking}

A total of 15 articles reported individual characteristics consistent with information-seeking behavior. Two articles were rated as weak methodologically, five articles as moderate-weak, and the remaining eight articles as moderate-strong (Additional Files 2 and 3). Sample sizes varied largely from a low of 92 participants [57] to a high of 5,948 participants [69] (Additional File 4). Several articles examined the relationships between different reading practices and research utilization. For example, reading professional journals [46]; hours spent reading professional journals $[44,47,48]$; the number of journals read [12,49,72]; and reading specific journals such as Heart and Lung [47,48], Nursing Research 
$[44,48]$, and $R N$ [44], were studied. Different combinations of these six reading characteristics were tested a total of 12 times (some articles assessed more than one of the reading practices simultaneously). Findings from these investigations were equivocal with seven articles (58\%) reporting statistically significant findings and five articles (42\%) not finding statistically significant findings. Thus, no conclusion can be drawn as to the effect of reading practices on nurses' use of research in practice.

The second most commonly studied information-seeking characteristic was attendance at conferences and/or attendance at in-services, examined in five articles [31,47-49,71]. Four of these articles [31,47-49], all rated moderate-strong with respect to methodological quality, found positive relationships, at statistically significant levels, between conference and/or in-service attendance and research utilization. The overall magnitude of this effect, however, is not computable since each article used a different test of statistical association. The remainder of individual characteristics falling within the category of information seeking were only investigated in one or two articles, precluding us from considering their findings (Table 1).

\section{Education}

A total of 28 articles reported individual characteristics within the domain of education, making it the most commonly studied category of characteristics in this review. Of the 28 articles, 10 were rated as weak methodologically, nine were rated as moderate-weak, and nine were rated as moderate-strong (Additional Files 2 and 3). Sample sizes varied from a low of 20 participants [54] to a high of 5,948 participants [69] (Additional File 4).

Twenty-five of the articles in this category examined one of three characteristics related to formal nursing education: increasing levels of education (i.e., diploma, bachelor degree, masters degree, $\mathrm{PhD}$ degree, but without post hoc analyses to determine between which levels noted differences lied), type of degree: bachelor versus diploma, and type of degree: graduate degree (masters or $\mathrm{PhD}$ ) versus lower (bachelor and/or diploma). Increasing levels of education was assessed in seven articles $[12,44,47,49,54,55,77]$. Findings from these investigations were equivocal with only four (57\%) of these articles $[12,49,54,77]$ finding positive relationships, at statistically significant levels, between higher levels of education and research utilization. A total of 11 articles $[14,29,31,46,57,61,62,69,71,73,76]$ examined the relationship between research utilization and type of degree: bachelor versus diploma. Eight of these articles $[14,31,46,57,61,62,73,76]$ did not find a significant association between bachelor degree versus diploma and research utilization, leading to the conclusion that type of degree: bachelor versus diploma is not an important characteristic to nurses' use of research. An additional seven articles (six studies) [29,33,34,48,50,59,60] examined the relationship between research utilization and type of degree: graduate degree (masters or $\mathrm{PhD}$ ) versus lower (bachelor and/or diploma). The majority of these articles $(n=5,71 \%)$ found a statistically significant relationship between graduate degree versus bachelor degree/diploma and research utilization $[29,33,34,48,50,59]$. Overall, findings from all 25 articles examining characteristics related to formal nursing education levels indicate that a positive effect exists for level of education, when a nurse holds a graduate degree compared to a bachelor degree/diploma but not when a nurse holds a bachelor degree compared to a diploma.

Another educational characteristic assessed in greater than four articles was completion of research classes $[12,30,44,47,55]$. Findings showed that this characteristic however was not significantly related to research utilization. Two articles [12,30], rated as weak and moderatestrong methodologically respectively, found a positive relationship, at statistically significant levels, while three articles $(60 \%)[44,47,55]$, one rated as weak methodologically and two rated as moderate-strong, did not find evidence of a statistically significant relationship.

The remaining individual characteristics related to education (e.g., well prepared in education process, working towards a degree, number of degrees, see Table 1 ) were assessed in less than four articles and therefore, were not considered.

\section{Professional characteristics}

The second most commonly studied category of individual characteristics, assessed in 27 of the 39 included articles, was professional characteristics. Of these articles, 12 were rated as weak methodologically, eight as moderate-weak, and eight as moderate-strong (Additional Files 2 and 3). Sample sizes varied from a low of 20 participants [54] to a high of 5,948 participants [69] (Additional File 4). The most commonly reported characteristics in this category were: experience (i.e., years employed as a nurse) ( $\mathrm{n}=12$ articles), current role (e.g., leadership compared to staff nurse) ( $\mathrm{n}=10$ articles), clinical specialty (e.g., critical care compared to medical/ surgical ( $n=9$ articles) and job satisfaction ( $n=5$ articles) (Table 1). Of these characteristics, consistent statistically significant relationships with research utilization were found for current role, specialty, and job satisfaction. Experience was not related to research utilization.

Ten articles (nine studies) examined the impact of current role on research utilization. Six (60\%) of these articles (three rated as weak methodologically, two as moderate-weak, and one as moderate-strong, see Additional Files 2 and 3 ) found that nurses practicing in 
advanced practice or leadership roles had significantly higher research utilization scores compared to staff nurses $[33,34,52,59,69,71]$. However, nurses in such advanced practice and leadership roles generally have higher levels of education, which may have confounded this finding. Nine articles examined the impact of clinical specialty on research utilization. Six (67\%) of these articles (two rated as weak, as moderate-weak, and as moderate-strong respectively, see Additional File 2) found a significant relationship between specialty and research utilization; nurses who worked on specialty wards (e.g., critical care, diabetes care) reported higher frequencies of research utilization in comparison to nurses who worked in more generalized units (e.g., medical or surgical floors) $[14,36,53,55,60,62]$. Five articles examined the impact of job satisfaction on research utilization. Three $(60 \%)$ of these articles (one rated as moderate-weak methodologically and two as moderatestrong, see Additional File 2) found a statistically significant relationship between job satisfaction and research utilization $[47,62,69]$. Experience, assessed in 12 articles, was not related to research utilization at statistically significant levels in the majority ( $\mathrm{n}=10$ of $12,83 \%)$ of these articles (Table 1).

\section{Socio-demographic and socio-economic factors}

Of the ten articles reporting other socio-demographic and socio-economic nurse characteristics (four rated as weak methodologically, three as moderate-weak, and three as moderate-strong, see Additional File 2), none reported a significant association with research utilization. Further, with the exception of age, which was assessed in nine studies, the characteristics were assessed in less than four studies, precluding the drawing of conclusions.

\section{Kinds of research utilization}

While the majority of articles identified in this review update assessed associations between individual characteristics and nurses' use of research in general, there is also a beginning trend in the literature to examine kinds of research utilization. A total of six articles (one rated as weak methodologically, two as moderate-weak, two as moderate-strong, and one as strong, see Additional File 2) were identified that explicitly examined the relationship between individual characteristics and nurses' use of one or more kinds of research utilization. The following section presents an overview of the findings from these six articles. More details on these findings can be found in Table 2.

The only individual characteristic assessed in a sufficient number of articles (i.e., in four or more articles) was a nurse's attitude towards research. All four articles reported a positive relationship, at statistically significant levels, between a nurse's attitude towards research and at least one kind of research utilization $[32,63,66,67]$. Only instrumental and overall kinds of research utilization were assessed in four articles. A positive relationship was found in three articles (75\%) for both of these kinds of research utilization: instrumental $[32,63,67]$ and overall $[32,66,67]$. All remaining characteristics were assessed in less than four articles, precluding conclusions.

One individual characteristic, critical thinking dispositions, was assessed in two articles examining kinds of research utilization. Critical thinking dispositions refers to a "set of attitudes that define a personal disposition to prize and to use critical thinking in one's personal, professional, and civic affairs" [79]. Both articles assessed critical thinking dispositions using the California Critical Thinking Disposition Inventory that measures seven dispositional components: truth-seeking, open-mindedness, analyticity, systematicity, self-confidence, inquisitiveness, and maturity [79]. Both identified studies found a positive relationship, at statistically significant levels, between nurses' ability to think critically (as measured by an average of all seven dispositions) and each of the four kinds of research utilization $[64,65]$. The magnitude of this effect was small to moderate with correlation coefficients ranging from 0.15 to 0.35 , depending on the kind of research utilization (Table 2).

\section{Discussion}

\section{Comparison with previous review}

This systematic review update focused on individual nurse characteristics that have been studied empirically with respect to nurses' use of research in practice. By extending the search criteria of the previous review, 31 additional studies were identified for inclusion in this update. This more than doubles the evidence available for review specifically examining the relationships between individual characteristics and research utilization by nurses. Unfortunately, studies continue to vary greatly in terms of sample selection (source of participants), sample size, study methods and rigor, statistical tests used, and the instrument (items) used to measure the outcome variable - research utilization. Promisingly, though, a trend in the most recent included studies for more robust analyses (i.e., multivariate regression versus bivariate correlations and/or tests of difference) and less variability in choice of outcome measures is evident. Nevertheless, given the continuing heterogeneity between studies, only general statements can be made regarding the relationships between individual characteristics and research utilization by nurses at this time. That is, at this point in time we can only say which characteristics are associated with research utilization 
and not which characteristics predict research utilization by nurses.

Taken collectively, the now significantly larger body of evidence suggests promise for the following individual characteristics as being important to (i.e., related to an increase in) nurses' use of research in their practice: positive attitude towards research, attending conferences and/or in-services, having a graduate degree (compared to a bachelors degree or diploma), current role (i.e., leadership and/or advanced practice compared to staff nurse), clinical specialty (working in critical care areas compared to general hospital units), and job satisfaction. An additional three characteristics were shown not to be important to research utilization by nurses: completion of research classes, experience, and age. While, overall, the extent to which many individual characteristics influence research utilization remains largely unknown, there is support for the above-mentioned characteristics. This represents a significant increase in knowledge over the previous review. Table 3 compares conclusions made in our review update with the original review.

\section{Kinds of research utilization}

In addition to examining the relationships between individual characteristics and research utilization generally, we also looked for relationships between individual characteristics and kinds (i.e., instrumental, conceptual, persuasive, and overall) of research utilization. Estabrooks [2] confirmed the existence of the four kinds of research utilization in a study of Canadian registered nurses, and additional studies since then have shown differential relationships between individual and contextual characteristics and the

Table 3 Comparison of conclusion between previous review and review update

\begin{tabular}{|c|c|c|c|}
\hline \multirow[t]{2}{*}{ Category } & \multirow{2}{*}{$\begin{array}{l}\text { Individual } \\
\text { Characteristic }\end{array}$} & \multicolumn{2}{|l|}{ Conclusion } \\
\hline & & $\begin{array}{l}\text { Previous Review } \\
\text { (Estabrooks et al., 2003) }\end{array}$ & Review Update \\
\hline \multirow[t]{2}{*}{ Beliefs and Attitudes } & $\begin{array}{l}\text { Attitude towards } \\
\text { research }\end{array}$ & $\begin{array}{l}\text { Positive attitude associated } \\
\text { with more research use }\end{array}$ & $\begin{array}{l}\text { Positive attitude associated with more research use (in general and } \\
\text { with instrumental and overall research utilization) }\end{array}$ \\
\hline & $\begin{array}{l}\text { All other } \\
\text { determinants }\end{array}$ & $\begin{array}{l}\text { No conclusion - Too few } \\
\text { studies }\end{array}$ & No conclusion - Too few studies \\
\hline \multirow[t]{2}{*}{$\begin{array}{l}\text { Involvement in Research } \\
\text { Activities }\end{array}$} & $\begin{array}{l}\text { Variety of } \\
\text { determinants }\end{array}$ & $\begin{array}{l}\text { No conclusion - Too few } \\
\text { studies }\end{array}$ & No conclusion - Too few studies \\
\hline & Reading practices & Equivocal & Equivocal \\
\hline \multirow[t]{2}{*}{ Information Seeking } & $\begin{array}{l}\text { Attending } \\
\text { conferences/in- } \\
\text { services }\end{array}$ & $\begin{array}{l}\text { No conclusion - Too few } \\
\text { studies }\end{array}$ & $\begin{array}{l}\text { Conference and/or in-service attendance associated with more } \\
\text { research use }\end{array}$ \\
\hline & $\begin{array}{l}\text { All other } \\
\text { determinants }\end{array}$ & $\begin{array}{l}\text { No conclusion - Too few } \\
\text { studies }\end{array}$ & No conclusion - Too few studies \\
\hline \multirow[t]{4}{*}{ Education } & Type of Degree & Equivocal & Bachelors versus diploma - no effect on research use \\
\hline & & & $\begin{array}{l}\text { Graduate versus bachelors/diploma - increased research use for } \\
\text { graduate degree }\end{array}$ \\
\hline & $\begin{array}{l}\text { Completion of } \\
\text { research classes }\end{array}$ & $\begin{array}{l}\text { No conclusion - Too few } \\
\text { studies }\end{array}$ & No association with research use \\
\hline & $\begin{array}{l}\text { All other } \\
\text { determinants }\end{array}$ & $\begin{array}{l}\text { No conclusion - Too few } \\
\text { studies }\end{array}$ & No conclusion - Too few studies \\
\hline \multirow[t]{5}{*}{ Professional Characteristics } & Years as an RN & $\begin{array}{l}\text { No association with research } \\
\text { use }\end{array}$ & No association with research use \\
\hline & Current role & $\begin{array}{l}\text { Leadership role associated } \\
\text { with more research use }\end{array}$ & Leadership role associated with more research use \\
\hline & Clinical specialty & $\begin{array}{l}\text { No association with research } \\
\text { use }\end{array}$ & $\begin{array}{l}\text { Working in critical care areas (compared to general wards) } \\
\text { associated with more research use }\end{array}$ \\
\hline & Job satisfaction & $\begin{array}{l}\text { No conclusion - Too few } \\
\text { studies }\end{array}$ & $\begin{array}{l}\text { Higher levels of job satisfaction associated with more research } \\
\text { use }\end{array}$ \\
\hline & $\begin{array}{l}\text { All other } \\
\text { determinants }\end{array}$ & $\begin{array}{l}\text { No conclusion - Too few } \\
\text { studies }\end{array}$ & No conclusion - Too few studies \\
\hline \multirow[t]{2}{*}{$\begin{array}{l}\text { Socio-Demographic and } \\
\text { Socio-Economic Factors }\end{array}$} & Age & $\begin{array}{l}\text { No association with research } \\
\text { use }\end{array}$ & No association with research use \\
\hline & $\begin{array}{l}\text { All other } \\
\text { determinants }\end{array}$ & $\begin{array}{l}\text { No conclusion - Too few } \\
\text { studies }\end{array}$ & No conclusion - Too few studies \\
\hline
\end{tabular}


different kinds of research utilization [32,63,66,67]. Therefore, we elected to report on these articles separately and not combine them with the articles that report on research utilization in general. While few articles were identified that have assessed relationships between individual characteristics and kinds of research utilization, some promising findings did emerge in those that were identified. For example, critical thinking, which was assessed in two articles showed positive, statistically significant correlations with each kind of research utilization in both articles $[64,65]$. These two articles were moderate-weak and moderate-high in methodological quality and had relatively small sample sizes of 143 and 287 nurses, respectively (Additional Files 2 and 4). This, combined with the limited number of studies conducted, precluded us from drawing a conclusion. While there is insufficient evidence at this time to conclude that a relationship does exist (and that nurses' critical thinking dispositions could be a target of future intervention studies), it may be a fruitful avenue for future research.

Despite a limited number of articles addressing kinds of research utilization, one characteristic - attitude towards research - was assessed in a sufficient number of articles (i.e., four articles) to be able to conclude a positive relationship between attitude towards research and nurses' instrumental and overall use of research exists. This relationship was also found in all eight articles examining attitude towards research on research utilization in general. This finding is consistent with known theories of human behavior. For example, the Theory of Planned Behavior, which is frequently used in psychological research, states human behavior (such as research utilization) is guided by three kinds of considerations: behavioral beliefs (i.e., beliefs about the likely outcomes of a behavior), normative beliefs (i.e., beliefs about the normative expectations of others and motivation to comply with these expectations), and control beliefs (i.e., beliefs about the presence of factors that may facilitate or impede performing the behavior) [80]. Behavioral beliefs are further known to produce a favorable or unfavorable attitude toward the behavior [80], supporting our findings.

Godin et al. [28], in a systematic review of healthcare professionals' (that included nurses) intentions and clinical behaviors, found the Theory of Planned Behavior to be an appropriate theory for examining attitudes and beliefs in relation to specific actions or behaviors. Specifically, they found healthcare professionals' beliefs about their own capabilities and the consequences of their behavior to be consistently and positively associated, at statistically significant levels, with predicting their clinical behavior. Beliefs were also positively and significantly associated with healthcare professionals' intention to change their behavior. These findings illustrate the potential benefit that using this theory, beyond the measurement of nurses' attitudes in general towards research utilization, may have in research utilization studies. For example, added value could be obtained by measuring nurses' beliefs and attitudes in relation to specific behaviors (i.e., their use of specific researchbased findings in practice). Future research should also focus on determining what causes nurses to form favorable (positive) attitudes towards the use of research, both of research utilization in general and of its kinds, as well as of the use of specific research-based findings in practice.

\section{Methodological implications for future research}

Systematic reviews typically identify and comment on problems with internal validity of the research under scrutiny, and this review update is no exception. Future studies examining individual characteristics related to research utilization need to attend to methodological quality to reduce bias and to increase confidence in this growing body of knowledge. This will allow for the design of theory-informed research utilization interventions with the intention of improving the quality of patient care.

Four important limitations of studies conducted to date on individual characteristics and research utilization by nurses are: methodological quality, statistical rigor, inconsistency in measurement of the outcome measure (research utilization), and limited use of research utilization or other related theory. First, few studies examining the relationship between individual characteristics and research utilization in this review were of moderate-strong or strong methodological quality, illustrating a clear need for well-designed, robust studies that examine the association between different individual characteristics and research utilization by nurses. Second, in order to effectively design research utilization interventions tailored for individual nurse characteristics, we need to know which characteristics predict (not just which ones are related to) research utilization. This will require multivariate statistical assessments. There is no need for continued bivariate assessments, especially given the clear evidence of inter-correlations among different individual characteristics [81]. Third, there is inconsistency in the measures being used for the outcome of interest, research utilization. By this we mean that we observed a lack of standard measures of research utilization across studies. While a few instruments that measure research use by nurses have been used in multiple studies (e.g., Nurses Practice Questionnaire [12,14,44-49], Research utilization Questionnaire [33,34,50-58]), Edmonton Research Orientation Survey [29,30,59], 
Estabrooks Kinds of Research Utilization Items [31,32,63-69]), by far, the most common approach to measuring research utilization has been the use of a single-item developed for an individual study. This absence of commonly used measures across studies makes it difficult, if not impossible, to build a consistent body of knowledge on which individual characteristics influence research utilization by nurses. Finally, only one-third $(\mathrm{n}=14$ ) of the articles identified in this review reported their investigation was based on research utilization or other appropriate theory (Additional File 4). For the vast majority of these articles, Rogers Diffusion of Innovations theory was used to guide the development of a measure of and/or calculation of a research utilization score, but not the selection of variables of included or the design and evaluation of the study. Future research utilization investigations should utilize appropriate theory in both instrument and study design/evaluation.

\section{Limitations}

While rigorous methods were used for this review, there were limitations. First, while an attempt was made to review grey literature (e.g., searching dissertation databases) we did not search all grey literature databases, and, as such, this review update may not be representative of all relevant work in the field. Second, where details of study methods were not clear, we did not attempt to clarify these details by contacting the article authors. This may have resulted in aspects of methods being scored low in the quality assessment phase, possibly reflecting quality of the reporting rather than the actual methods used. Third, studies published in languages other than those of the research team were excluded. Finally, because of the inconsistency in how associations between individual characteristics and research utilization were determined and reported in the included studies, we were forced to use a vote-counting approach to data synthesis. There are several weaknesses associated with using vote counting. For example, this approach to synthesis fails to account for: effect sizes (vote counting gives equal weight to all associations, regardless of magnitude) and precision of the estimate from the primary studies (vote counting gives equal weight to comparisons irrespective of sample size). To lessen these problems, we reported the following as recommended by Grimshaw et al. [26]: all associations showing a positive direction of effect, the number of comparisons showing statistically significant effects (regardless of direction), and the magnitude of effect for significant findings when it was provided in the articles.

\section{Conclusion}

This review update points to an increased body of research on the study of individual characteristics and research utilization by nurses. However, methodological problems inherent in many of the studies included in the review update mean that robust evidence to support individual characteristics that predict research utilization is scarce. Current evidence suggests that a nurse's attitude towards research is the only individual characteristic that is consistently (with a positive effect) related to research utilization in general and the different kinds of research utilization. Other individual characteristics with evidence for a positive association with research utilization (in general) include: attending conferences and/or in-services, having a graduate degree, current role, clinical specialty, and job satisfaction. These characteristics may hold promise as targets of future research utilization interventions. While all of these characteristics are potentially modifiable, some can be more easily manipulated and thus incorporated into interventions to increase research utilization. For example, attitude towards research and attendance at conferences and/or in-services are two characteristics that we believe can and should be the focus of future research utilization interventions. The remaining characteristics identified in this review as having a positive statistically significant association with research utilization, while modifiable, would require more effort and time to implement (e.g., increasing the number of nurses employed within a clinical setting that hold a graduate degree).

We also recommend that programmatic research in the area of research utilization in nursing be undertaken. Programmatic research differs from conducting a research study in that it seeks to break a large research topic into smaller, more manageable pieces, allowing for more detailed analyses. Importantly, programmatic research addresses each piece sequentially in an effort to build a coherent picture from the smaller studies' findings, and allows investigators to build upon their own and others' research. Such programs in research utilization in nursing would have several concurrent streams examining, for example, different settings (acute care adults, acute care pediatrics, long-term care, community/home healthcare), different classes of determinants (individual characteristics, contextual factors, and organizational factors), and interventions to increase research use and subsequently patient outcomes. Without such programmatic research, we believe substantial advances in understanding how to increase the use of research by nurses and thereby improve patient care will be difficult, if not impossible, to achieve. 


\section{Additional material}

Additional file 1: Search strategy. A summary of the search strategy used in the review.

Additional file 2: Quality assessment for included cross sectional articles. A description of the findings from the quality assessment of included articles describing studies that used a cross sectional study design.

Additional file 3: Quality assessment for the included quasiexperimental articles. A description of the findings from the quality assessment of included articles describing studies that used a quasiexperimental study design.

Additional file 4: Characteristics of the included studies. A detailed summary of the characteristics of all articles included in the review.

\section{Acknowledgements}

This project was made possible by the support of the Canadian Institutes of Health Research (CIHR) Knowledge Translation Synthesis Program (KRS 86255). JES holds CIHR Postdoctoral and Bisby Fellowships; at the time of this research she held Killam, CIHR and Alberta Heritage Foundation for Medical Research (AHFMR) doctoral fellowships. CAE holds a CIHR Canada Research Chair in Knowledge Translation. PG holds a grant from AFA Insurance and LW is supported by the Center for Care Sciences at Karolinska Institutet.

We would like to thank the following individuals for their contribution to the project reported in this paper: Dagmara Chojecki, MLIS, for her support in finalizing the search strategy and Hannah O'Rourke, research assistant, for her assistance with screening and quality assessment.

\section{Author details}

${ }^{1}$ Clinical Epidemiology Program, Ottawa Hospital Research Institute, Ottawa, Ontario, Canada. ${ }^{2}$ Faculty of Nursing, University of Alberta, Edmonton, Alberta, Canada. ${ }^{3}$ Division of Psychology, Department of Clinical Neuroscience, Karolinska Institutet, Stockholm, Sweden. ${ }^{4}$ Division of Nursing, Department of Neurobiology, Care Sciences and Society, Karolinska Institutet; and Clinical Research Utilization (CRU), Karolinska University Hospital, Stockholm, Sweden.

\section{Authors' contributions}

All authors participated in designing the study, securing funding for the project, and developing the search strategy. JES undertook the article selection; data extraction and quality assessment; and drafted the manuscript. CAE, PG, and LW provided valuable advice throughout the study. All authors provided critical commentary on the manuscript and approved the final version.

\section{Competing interests}

The authors declare that they have no competing interests.

Received: 22 March 2010 Accepted: 5 January 2011

Published: 5 January 2011

\section{References}

1. Estabrooks CA, Wallin L, Milner M: Measuring knowledge utilization in health care. International Journal of Policy Analysis \& Evaluation 2003, 1:3-36.

2. Estabrooks CA: The conceptual structure of research utilization. Research in Nursing and Health 1999, 22(3):203-216.

3. Stetler C: Research utilization: Defining the concept. Image:The Journal of Nursing Scholarship 1985, 17:40-44.

4. Estabrooks CA: Will Evidence-Based Nursing Practice Make Practice Perfect? Canadian Journal of Nursing Research 1998, 30(1):15-36.

5. English I: Nursing as a research based profession: 22 years after Briggs. British Journal of Nursing 1994, 3(8):402-406.

6. Dobbins M, Ciliska D, Mitchell A: Dissemination and use of research evidence for policy and practice by nurses: A model of development and implementation strategies. PhD Thesis University of Toronto: Faculty of Nursing; 1998.

7. Veeramah $\mathrm{V}$ : Utilization of research findings by graduate nurses and midwives. Journal of Advanced Nursing 2004, 47(2):183-191.

8. Larsen K, Adamsen L, Bjerregaard L, Madsen L: There is no gap 'per se' between theory and practice: Research knowledge and clinical knowledge are developed in different contexts and follow their own logic. Nursing Outlook 2002, 50(5):204-212.

9. Allmark P: A classical view of the theory-practice gap in nursing. Journal of Advanced Nursing 1995, 22(1):18-23.

10. Fealy G: The theory-practice relationship in nursing: An exploration of contemporary discourse. Journal of Advanced Nursing 1997, 25(5):1061-1069.

11. Bircumshaw D: The utilization of research findings in clinical nursing practice. Journal of Advanced Nursing 1990, 15:1272-1280.

12. Rodgers SE: The extent of nursing research utilization in general medical and surgical wards. Journal of Advanced Nursing 2000, 32(1):182-193.

13. Parahoo K: Barriers to, and facilitators of, research utilization among nurses in Northern Ireland. Journal of Advanced Nursing 2000, 31(1):89-98.

14. Squires JE, Moralejo D, LeFort SM: Exploring the role of organizational policies and procedures in promoting research utilization in registered nurses. Implementation Science 2007, 2(1).

15. Wallin L: Knowledge translation and implementation research in nursing. International Journal of Nursing Studies 2009, 46:576-587.

16. Thompson DS, Estabrooks CA, Scott-Findlay S, Moore K, Wallin L, Thompson DS, Estabrooks CA, Scott-Findlay S, Moore K, Wallin L: Interventions aimed at increasing research use in nursing: a systematic review. Implementation Science 2007, 2:15.

17. Estabrooks CA, Floyd JA, Scott-Findlay S, O'Leary KA, Gushta M: Individual determinants of research utilization: A systematic review. Journal of Advanced Nursing 2003, 43(5):506-520.

18. Squires J, Estabrooks C, Wallin L, Gustavsson P: A systematic review of the psychometric properties of instruments used to measure knowledge translation in healthcare professionals technical report. Edmonton, Alberta: Faculty of Nursing, University of Alberta; 2009.

19. De Vet HCW, De Bie RA, Van Der Heijden GJMG, Verhagen AP, Sijpkes $P$, Knipschild PG: Systematic reviews on the basis of methodological criteria. Physiotherapy 1997, 83(6):284-289.

20. Cummings $G$, Estabrooks $C$ : The effects of hospital restructuring that included layoffs on individual nurses who remained employed: A systematic report of impact. International Journal of Sociology and Social Policy 2003, 8:8-53.

21. Estabrooks C, Cummings G, Olivo S, Squires J, Gibin C, Simpson N: Effects of shift length on quality of patient care and health provider outcomes: systematic review. Quality and Safety in Healthcare 2009, 18:181-188.

22. Khan K, Ter Riet G, Popay J, Nixon J, Kleijnen J, Satge I: Conducting the review: Phase 5 study quality assessment. Undertaking systematic reviews of research effectiveness CDC's guidance for those carrying out or commissioning reviews Centre of Reviews and Dissemination UoY; 2001, $1-20$.

23. Kmet $L$, Lee $R$, Cook L: Standard quality assessment criteria for evaluating primary research papers from a variety of fields. Edmonton: Alberta Heritage Foundation for Medical Research; 2004, 1-22.

24. Jackson N, Waters E: Criteria for the systematic review of health promotion and public health interventions. Health Promotion International 2005, 20(4):367-374.

25. Deeks JJ, Dinnes J, D'Amico R, Sowden AJ, Sakarovitch C, Song F Petticrew M, Altman DG: Evaluating non-randomised intervention studies. Health Technology Assessment (Winchester, England) 2003, 7(27).

26. Grimshaw J, McAuley LM, Bero LA, Grilli R, Oxman AD, Ramsay C, Vale L, Zwarenstein M: Systematic reviews of the effectiveness of quality improvement strategies and programmes. Quality \& Safety in Health Care 2003, 12(4):298-303.

27. Levels of Evidence. [http://consumers.cochrane.org/levels-evidence]

28. Godin G, Belanger-Gravel A, Eccles M, Grimshaw G: Healthcare professionals' intentions and behaviours: A systematic review of studies based on social cognitive theories. Implementation Science 2008, 3(36).

29. McCleary L, Brown GT: Use of the Edmonton research orientation scale with nurses. Journal of Nursing Measurement 2002, 10(3):263-275. 
30. McCleary L, Brown GT: Association between nurses' education about research and their reseach use. Nurse Education Today 2003, 23(8):556-565.

31. Estabrooks CA: Modeling the individual determinants of research utilization. Western Journal of Nursing Research 1999, 21(6):758-772.

32. Estabrooks CA, Kenny DJ, Adewale AJ, Cummings GG, Mallidou AA: A comparison of research utilization among nurses working in Canadian civilian and United States Army healthcare settings. Research in Nursing and Health 2007, 30(3):282-296.

33. McCloskey DJ: The relationship between organizational factors and nurse factors affecting the conduct and utilization of nursing research. PhD Thesis George Mason University; 2005.

34. McCloskey DJ, McCloskey DJ: Nurses' perceptions of research utilization in a corporate health care system. Journal of Nursing Scholarship 2008, 40(1):39-45.

35. Parahoo K: A comparison of pre-Project 2000 and Project 2000 nurses' perceptions of their research training, research needs and of their use of research in clinical areas. Journal of Advanced Nursing 1999, 29(1):237-245.

36. Parahoo K, McCaughan EM: Research utilization among medical and surgical nurses: A comparison of their self reports and perceptions of barriers and facilitators. Journal of Nursing Management 2001, 9(1):21-30.

37. Bostrom J, Suter WN: Research utilization: making the link to practice. Journal of Nursing Staff Development 1993, 9(1):28-34.

38. Kirchhoff KT: A diffusion survey of coronary precautions. Nursing Research 1982, 31(4):196-201.

39. Davies BL: Evaluation of two strategies for the transfer of research results about labour support and electronic fetal monitoring into practice. Dissertation/Thesis University of Toronto (Canada); 1999.

40. Winter JC: Brief: relationship between sources of knowledge and use of research findings. Journal of Continuing Education in Nursing 1990, 21(3):138-140.

41. Lia-Hoagberg B, Schaffer M, Strohschein S: Public health nursing practice guidelines: an evaluation of dissemination and use. Public Health Nursing 1999, 16(6):397-404.

42. Brett JL: Organizational integrative mechanisms and adoption of innovations by nurses. Nursing Research 1989, 38(2):105-110.

43. Parahoo K: Research utilization and attitudes towards research among psychiatric nurses in Northern Ireland. Journal of Psychiatric and Mental Health Nursing 1999, 6(2):125-135.

44. Brett JL: Use of nursing practice research findings. Nursing Research 1987, 36(6):344-349

45. Barta KM: Information-seeking, research utilization, and barriers to research utilization of pediatric nurse educators. Journal of professional nursing: official journal of the American Association of Colleges of Nursing 1995, 11(1):49-57.

46. Berggren A: Swedish midwives' awareness of, attitudes to and use of selected research findings. Journal of Advanced Nursing 1996. 23(3):462-470

47. Coyle LA, Sokop AG: Innovation adoption behavior among nurses. Nursing Research 1990, 39(3):176-180.

48. Michel $Y$, Sneed NV: Dissemination and use of research findings in nursing practice. Journal of Professional Nursing 1995, 11(5):306-311.

49. Rutledge DN, Greene P, Mooney K, Nail LM, Ropka M: Use of researchbased practices by oncology staff nurses. Oncology Nursing Forum 1996, 23(8):1235-1244

50. Champion $\mathrm{VL}$, Leach $\mathrm{A}$ : Variables related to research utilization in nursing: an empirical investigation. Journal of Advanced Nursing 1989, 14(9):705-710.

51. Bostrom AM, Kajermo KN, Nordstrom G, Wallin L: Barriers to research utilization and research use among registered nurses working in the care of older people: Does the BARRIERS Scale discriminate between research users and non-research users on perceptions of barriers? Implementation Science 2008, 3(1)

52. Hatcher S, Tranmer J: A survey of variables related to research utilization in nursing practice in the acute care setting. Canadian Journal of Nursing Administration 1997, 10(3):31-53.

53. Humphris D, Hamilton S, O'Halloran P, Fisher S, Littlejohns P: Do diabetes nurse specialists utilise research evidence? Practical Diabetes International 1999, 16(2):47-50.

54. Lacey EA: Research utilization in nursing practice - a pilot study. Journal of Advanced Nursing 1994, 19(5):987-995.
55. Nash MA: Research utilization among Idaho nurses. PhD Thesis Gonzaga University; 2005.

56. Prin PL, Mills MD, Gerdin U: Nurses' MEDLINE usage and research utilization. In Nursing informatics: the impact of nursing knowledge on health care informatics proceedings of N'97, Sixth Triennial International Congress of IMIA-NI, Nursing Informatics of International Medical Informatics Association. Volume 46. Amsterdam, Netherlands: IOS Press; 1997:451-456.

57. Tranmer JE, Lochhaus-Gerlach J, Lam M: The effect of staff nurse participation in a clinical nursing research project on attitude towards, access to, support of and use of research in the acute care setting. Canadian Journal of Nursing Leadership 2002, 15(1):18-26.

58. Wallin L, Bostrom A, Wikblad K, Ewald U: Sustainability in changing clinical practice promotes evidence-based nursing care. Journal of Advanced Nursing 2003, 41(5):509-518.

59. Bonner A, Sando J: Examining the knowledge, attitude and use of research by nurses. Journal of Nursing Management 2008, 16(3):334-343.

60. Stiefel KA: Career commitment, nursing unit culture, and nursing research utilization. Dissertation/Thesis University of South Carolina; 1996.

61. Varcoe C, Hilton A: Factors affecting acute-care nurses' use of research findings. Canadian Journal of Nursing Research 1995, 27(4):51-71.

62. Forbes SA, Bott MJ, Taunton RL: Control over nursing practice: a construct coming of age. Journal of Nursing Measurement 1997, 5(2):179-190.

63. Kenny DJ: Nurses' use of research in practice at three US Army hospitals. Canadian journal of nursing leadership 2005, 18(3):45-67.

64. Profetto-McGrath J, Hesketh KL, Lang S, Estabrooks CA: A study of critical thinking and research utilization among nurses. West J Nurs Res 2003, 25(3):322-337.

65. Profetto-McGrath J, Smith KB, Hugo K, Patel A, Dussault B: Nurse educators' critical thinking dispositions and research utilization. Nurse Education in Practice 2009, 9(3):199-208.

66. Connor N: The relationship between organizational culture and research utilization practices among nursing home departmental staff. MN Thesis Dalhousie University (Canada); 2007

67. Milner FM, Estabrooks CA, Humphrey C: Clinical nurse educators as agents for change: increasing research utilization. International Journal of Nursing Studies 2005, 42(8):899-914.

68. Cummings GG, Estabrooks CA, Midodzi WK, Wallin L, Hayduk L: Influence of organizational characteristics and context on research utilization. Nurs Res 2007, 56(4 Suppl):S24-39.

69. Wallin L, Estabrooks CA, Midodzi WK, Cummings GG: Development and validation of a derived measure of research utilization by nurses. Nursing Research 2006, 55(3):149-160

70. Brown DS: Nursing education and nursing research utilization: is there a connection in clinical settings? Journal of Continuing Education in Nursing 1997, 28(6):258-262, quiz 284

71. Butler L: Valuing research in clinical practice: a basis for developing a strategic plan for nursing research. The Canadian journal of nursing research 1995, 27(4):33-49.

72. Wells N, Baggs JG: A survey of practicing nurses' research interests and activities. Clinical Nurse Specialist: The Journal for Advanced Nursing Practice 1994, 8(3):145-151.

73. Ofi B, Sowunmi L, Edet D, Anarado N, Ofi B, Sowunmi L, Edet D, Anarado N: Professional nurses' opinion on research and research utilization for promoting quality nursing care in selected teaching hospitals in Nigeria. International Journal of Nursing Practice 2008, 14(3):243-255.

74. Tsai S: Nurses' participation and utilization of research in the Republic of China. International Journal of Nursing Studies 2000, 37(5):435-444.

75. Tsai S: The effects of a research utilization in-service program on nurses. International Journal of Nursing Studies 2003, 40(2):105-113.

76. Erler CJ, Fiege AB, Thompson CB: Flight nurse research activities. Air Medical Journal 2000, 19(1):13-18.

77. Logsdon C, Davis DW, Hawkins B, Parker B, Peden A: Factors related to research utilization by registered nurses in Kentucky. Kentucky Nurse 1998, 46(1):23-26.

78. Wright A, Brown P, Sloman R: Nurses' perceptions of the value of nursing research for practice. Australian Journal of Advanced Nursing 1996, 13(4):15-18.

79. Facione N, Facione P, Sanchez C: Critical thinking disposition as a measure of competent clinical judgement: The development of the California Critical Thinking Disposition Inventory. Journal of Nursing Education 1994, 33(8):345-350. 
80. Ajzen I: The theory of planned behavior. Organizational Behavior and Human Decision Processes 1991, 50:179-211.

81. Estabrooks C: A program of research in knowledge translation. Nursing Research 2007, 56(Supplement):S4-S5.

doi:10.1186/1748-5908-6-1

Cite this article as: Squires et al: Individual determinants of research utilization by nurses: a systematic review update. Implementation Science 2011 6:1.

Submit your next manuscript to BioMed Central and take full advantage of:

- Convenient online submission

- Thorough peer review

- No space constraints or color figure charges

- Immediate publication on acceptance

- Inclusion in PubMed, CAS, Scopus and Google Scholar

- Research which is freely available for redistribution 